\title{
Stenotrophomonas maltophilia responds to exogenous AHL signals through the LuxR solo SmoR (Smlt1839)
}

\section{OPEN ACCESS}

Edited by:

Vittorio Venturi,

International Centre for Genetic Engineering and Biotechnology, Italy

Reviewed by:

Andre O. Hudson,

Rochester Institute of Technology,

USA

Anice Sabag-daigle

The Ohio State University, USA Bruna Gonçalves Coutinho,

University of Washington, USA

${ }^{*}$ Correspondence:

Daniel Yero and Isidre Gibert, Departament de Genètica i de

Microbiologia, Institut de

Biotecnologia i de Biomedicina, Universitat Autònoma de Barcelona,

Campus Universitari, Bellaterra

08193, Barcelona, Spain daniel.yero@uab.cat; isidre.gibert@uab.cat

${ }^{t}$ These authors have contributed equally to this work.

Received: 29 January 2015 Accepted: 28 April 2015 Published: 15 May 2015

Citation:

Martínez P, Huedo P, Martínez-Servat S, Planell R, Ferrer-Navarro M, Daura

$X$, Yero D and Gibert I (2015)

Stenotrophomonas maltophilia responds to exogenous $A H L$ signals through the LuxR solo SmoR

(Smlt1839).

Front. Cell. Infect. Microbiol. 5:41. doi: 10.3389/fcimb.2015.00041

\begin{abstract}
Paula Martínez ${ }^{1,2 \dagger}$, Pol Huedo ${ }^{1,2 \dagger}$, Sònia Martinez-Servat ${ }^{1,2}$, Raquel Planell, Mario Ferrer-Navarro ${ }^{1}$, Xavier Daura ${ }^{1,3}$, Daniel Yero ${ }^{1,2 *}$ and Isidre Gibert ${ }^{1,2 *}$
\end{abstract}

\footnotetext{
1 Grup de Genètica Molecular i Patogènesi Bacteriana, Institut de Biotecnologia i de Biomedicina, Universitat Autònoma de Barcelona, Barcelona, Spain, ${ }^{2}$ Departament de Genètica i de Microbiologia, Universitat Autònoma de Barcelona, Barcelona, Spain, ${ }^{3}$ Catalan Institution for Research and Advanced Studies, Barcelona, Spain
}

Quorum Sensing (QS) mediated by Acyl Homoserine Lactone (AHL) molecules are probably the most widespread and studied among Gram-negative bacteria. Canonical AHL systems are composed by a synthase (Luxl family) and a regulator element (LuxR family), whose genes are usually adjacent in the genome. However, incomplete AHL-QS machinery lacking the synthase Luxl is frequently observed in Proteobacteria, and the regulator element is then referred as LuxR solo. It has been shown that certain LuxR solos participate in interspecific communication by detecting signals produced by different organisms. In the case of Stenotrophomonas maltophilia, a preliminary genome sequence analysis revealed numerous putative luxR genes, none of them associated to a luxl gene. From these, the hypothetical LuxR solo Smlt1839, here designated SmoR, presents a conserved AHL binding domain and a helix-turn-helix DNA binding motif. Its genomic organization-adjacent to hchA gene-indicate that SmoR belongs to the new family "LuxR regulator chaperone HchA-associated." AHL-binding assays revealed that SmoR binds to AHLs in-vitro, at least to oxo-C8-homoserine lactone, and it regulates operon transcription, likely by recognizing a conserved palindromic regulatory box in the hchA upstream region. Supplementation with concentrated supernatants from Pseudomonas aeruginosa, which contain significant amounts of AHLs, promoted swarming motility in S. maltophilia. Contrarily, no swarming stimulation was observed when the $P$. aeruginosa supernatant was treated with the lactonase AilA from Bacillus subtilis, confirming that AHL contributes to enhance the swarming ability of $S$. maltophilia. Finally, mutation of $s m o R$ resulted in a swarming alteration and an apparent insensitivity to the exogenous AHLs provided by $P$. aeruginosa. In conclusion, our results demonstrate that $S$. maltophilia senses AHLs produced by neighboring bacteria through the LuxR solo SmoR, regulating population behaviors such as swarming motility.

Keywords: LuxR Orphan, AHL, Acyl-Homoserine lactone, lactonase, quorum sensing, swarming 


\section{Introduction}

Bacterial cells can communicate with each other to facilitate their rapid adaptation to fluctuations in the environment. This cell-cell communication mechanism, known as quorum sensing (QS), relies primarily on the production, detection, and response to diffusible signal molecules (also called autoinducers) in a cell-density dependent manner (Fuqua et al., 1994; Whitehead et al., 2001; Fuqua and Greenberg, 2002; Federle and Bassler, 2003). Through this QS communication, numerous bacterial species regulate a variety of functions such as biofilm formation, motility, antibiotic resistance, toxin production, exopolysaccharide synthesis, and extracellular enzyme production among others (Miller and Bassler, 2001). In Gram-negative bacteria, $N$-acyl homoserine lactones (AHLs) are to date the most extensively and best characterized QS signaling molecules. AHL-QS regulation consists of a LuxI-type synthase, which produces signal molecules, and a LuxR-type receptor that binds AHLs and regulates expression of certain genes when signal concentration reaches a critical threshold. LuxR regulators are about 250 residues in length and present two typical domains, the $\mathrm{N}$-terminal autoinducer AHL binding domain (Shadel et al., 1990; Slock et al., 1990) and the C-terminal helix-turn-helix (HTH) DNA-binding domain (Choi and Greenberg, 1991; Fuqua and Winans, 1994). In the presence of AHLs, the N-terminal binding domain interacts with the signal molecule, habilitating the DNA-binding domain to induce transcription of certain genes by binding to their promoters in a region named luxR box (Devine et al., 1989; Stevens and Greenberg, 1997). The DNAbinding domain includes three highly conserved aminoacids, while the AHL-binding domain presents six hydrophobic or aromatic residues displaying remarkable variability (18-25\%) (Zhang et al., 2002).

The increasing availability of bacterial genome sequences has led to the identification of several LuxR and LuxI homologs. Typically, both luxI-type and luxR-type genes are located adjacent in the bacterial genome (the cognate luxR/I pair). However, luxR-type genes without a cognate luxI-type in their vicinity are frequently found, and these regulatory elements are then called "orphan" (Fuqua, 2006) or "solo" LuxR (Subramoni and Venturi, 2009a). Recent studies have revealed that "luxR solo" genes are widely distributed among bacterial genomes (Hudaiberdiev et al., 2015). LuxR solos present the same modular organization as canonical LuxR, displaying the $\mathrm{N}$-terminal and C-terminal domains. The nature of the signal molecules that bind to the different LuxR solos is quite heterogeneous. It has been shown that the LuxR solo QscR from Pseudomonas aeruginosa bind to self-produced AHL signals (Chugani et al., 2001; Lequette et al., 2006), while SdiA of Salmonella enterica and Escherichia coli respond to exogenous AHL signals (Ahmer et al., 1998; Michael et al., 2001; Ahmer, 2004; Yao et al., 2006). Interestingly, the LuxR solo OryR from Xanthomonas oryzae pv. oryzae interacts with plant signals, in particular those produced by rice (Ferluga et al., 2007; Ferluga and Venturi, 2009; González et al., 2013). More recently, it has been reported that the human and insect pathogen Photorhabdus asymbiotica contains a LuxR solo PauR that is the regulator element of a new QS-system, which is mediated by dialkylresorcinols (DARs) and cyclohexanediones (CHDs) signals (Brameyer et al., 2015). Altogether, this shows that LuxR solos can participate in a wide variety of signaling networks.

Stenotrophomonas maltophilia is an ubiquitous gramnegative bacterium considered an emerging nosocomial pathogen (Brooke, 2012). Moreover, it is frequently found in lungs of cystic-fibrosis (CF) patients (Demko et al., 1998), usually co-isolated with $P$. aeruginosa (Moskowitz et al., 2005). The QS described in S. maltophilia is based on the signaling molecule DSF (11-cis-2-decenoic acid), by which it regulates virulence-related processes (Fouhy et al., 2007; Huedo et al., 2014). To date, no S. maltophilia strain has been reported to produce AHL and the K279a reference genome contains no luxI homolog (Crossman et al., 2008), at least of the usual luxI types (Waters and Bassler, 2005). However, sequence analysis reveals that this genome encodes a total of 15 putative LuxR-like proteins, based mainly on homologies of the DNA-binding response domain. From these, only the LuxR solo Smlt1839 showed an N-AHL autoinducer-binding domain. The objective of our study has been to experimentally investigate the role of Smlt1839 in AHL binding and swarming regulation in S. maltophilia, in the presence of exogenous and heterologous AHLs.

\section{Materials and Methods}

\section{Bacterial Strains and Growth Conditions}

Bacterial strains used in this study are listed in Table 1. E. coli strains DH5a and BL21 (DE3) were used for general cloning purposes and overexpression of $s m o R$, respectively. S. maltophilia E77 (Ferrer-Navarro et al., 2013) was used as a model strain to investigate the role of SmoR in detection and response of exogenous AHL signals. P. aeruginosa MPAO1 strain was used as an AHL-producer bacterium to evaluate the effect of exogenous signal molecules on swarming motility of $S$. maltophilia. Agrobacterium tumefaciens KYC55 (Zhu et al., 2003) was used as a reporter strain to detect AHL production.

E. coli, S. maltophilia and $P$. aeruginosa strains were routinely grown in Luria-Bertani (LB) medium at $37^{\circ} \mathrm{C}$. A. tumefaciens KYC55 was grown in AT medium (Fuqua and Winans, 1994) at $30^{\circ} \mathrm{C}$. When required, the antibiotics were supplemented as follows: ampicillin (Ap) $20 \mu \mathrm{g} / \mathrm{ml}$ (E. coli); tetracycline (Tc) $17 \mu \mathrm{g} / \mathrm{ml}$ (E. coli and P. aeruginosa) or $2 \mu \mathrm{g} / \mathrm{ml}$ (A. tumefaciens); erythromycin (Erm) $50 \mu \mathrm{g} / \mathrm{ml}$ (E. coli) or $500 \mu \mathrm{g} / \mathrm{ml}$ (S. maltophilia); gentamicin (Gm) $10 \mu \mathrm{g} / \mathrm{ml}$ (E. coli), $100 \mu \mathrm{g} / \mathrm{ml}$ (A. tumefaciens) or $40 \mu \mathrm{g} / \mathrm{ml}$ (S. maltophilia); and spectinomycin (Spc) $100 \mu \mathrm{g} / \mathrm{ml}$ (E. coli and A. tumefaciens).

\section{Sequence Determination and In Silico Analysis}

A $5.5 \mathrm{~kb}$ fragment containing the ORFs of smlt1840 and smlt1839 plus their flanking regions was amplified from S. maltophilia strain E77 genomic DNA and subsequently sequenced (Macrogen). The sequence has been used for sequence alignments as well as reference to generate a $\Delta s m o R$ mutant in this model strain. The fragment corresponding to hchA-smoR operon and its predicted promoter (1658 bp) was submitted to 
TABLE 1 | Primers used in this study.

\begin{tabular}{llc}
\hline Primer & Sequence $\left(\mathbf{5}^{\prime} \mathbf{- 3}^{\prime} \mathbf{)}\right.$ & Restriction site \\
\hline P1MutSmoR & AAGCTTGCCCGGTCGGTATCGG & Hindlll \\
P2MutSmoR & GGATCCTCGCGGCGAGGCACTCC & BamHl \\
P3MutSmoR & GGATCCCCGGTCAGCGCCCGGCC & BamHl \\
P4MutSmoR & GAATTCCCAGCCGCCAGCCCAGC & EcoRl \\
PErm5' & GGATCCGAAACGTAAAAGAAGTATG & BamHl \\
PErm3' & GGATCCTACAAATTCCCCGTAGGC & BamHl \\
PErm5'rev & GATACTGCACTATCAACACAC & - \\
PErm3'rev & CTTCCAAGGAGCTAAAGAGGT & - \\
P1DemSmoR & GTACGTCGGGCGTATCG & - \\
P2DemSmoR & GCCCTTCTATGCTGG & - \\
P1ProSmoR & TCTAGACGCACACGCATGGACCG & Xbal \\
P2ProSmoR & GGATCCGAAGGCGTCGCGCTCGG & BamHl \\
P1ExpSmoR & CATATGAGCGATCTGGTGCAGGCG & Ndel \\
P2ExpSmoR & CTCGAGTCAGTCTCGATCTCGCCT & Xhol \\
PT7up & TAATACGACTCACTATAGGG & - \\
PT7dw & GCTAGTATTGCTCAGCGG & - \\
\hline
\end{tabular}

Genbank under the accession number KP691985. Annotation was done using BLAST (Altschul et al., 1990) and intergenic regions were manually inspected for palindromic motifs and cis elements that participate in regulating translation. Program RSAT (Thomas-Chollier et al., 2011) was used to scan for a pattern (the palindromic box) within all ORF upstream regions in the $\mathrm{K} 279 \mathrm{a}$ genome. A simple screen for luxR-like genes using a S. maltophilia K279a genomic sequence (AM743169.1) was done by using BLAST and PSI-BLAST (Altschul et al., 1997) to detect remote homologs. Sequences of the hchAsmoR operon from other $S$. maltophilia strains were retrieved from their genome sequences at NCBI (http://www.ncbi.nlm. nih.gov/genome/). Translation of ORFs to amino-acid sequences and sequence alignments were done with MEGA 6 (Tamura et al., 2013) and then analyzed with SMART (Letunic et al., 2009) for the identification and annotation of protein domains. Nucleotide and protein sequences were aligned using the ClustalW module implemented in MEGA 6 and manually edited and visualized with BioEdit. Software was run with default parameters unless otherwise stated. Identification of "LuxR-like regulators chaperone HchA associated" in other Proteobacteria was predicted by InterPro (http://www.ebi.ac.uk/interpro/) (Mitchell et al., 2014).

\section{Preparation of Fusion and Expression Vectors}

Oligonucleotides used as primers and plasmids used in this study are listed in Tables 2 and 3, respectively. The transcriptional fusion construct for the $s m o R$ promoter in pBBR1MCS-5-lacZ (Fried et al., 2012) was generated by amplifying a fragment of 415 bp containing the putative promoter of the operon hchA-smoR (smlt1840-smlt1839) from S. maltophilia E77, using primers P1ProSmoR and P2ProSmoR and FastStart DNA polymerase (Roche). The fragment was digested using XbaI and BamHI and cloned into their respective restriction sites into pBBR5MCS5-lacZ, generating pBBR5MCS-PsmoR::LacZ. This vector was
TABLE 2 | Plasmids used in this study.

\begin{tabular}{|c|c|c|}
\hline Plasmid & Relevant Characteristics & Source \\
\hline pGEM-Erm & $\begin{array}{l}\text { Cloning vector carrying Erm resistance } \\
\text { gene, } A m p^{r}, \mathrm{Erm}^{r}\end{array}$ & This work \\
\hline pEX18Tc & Suicide allelic exchange vector; $T c^{r}$ & $\begin{array}{l}\text { Hoang et al., } \\
1998\end{array}$ \\
\hline pEXsmoR & $\begin{array}{l}\text { pEX18Tc carrying E77 smoR flanking } \\
\text { regions interrupted with Erm resistance } \\
\text { gene, } T^{r}{ }^{r}, E r m^{r}\end{array}$ & This work \\
\hline pBBR1MCS-5 & Broad-host-range cloning vector, $\mathrm{Gm}^{r}$ & $\begin{array}{l}\text { Kovach et al., } \\
1995\end{array}$ \\
\hline pET22b & IPTG inducible expression vector, $A m p^{r}$ & Novagen \\
\hline pET22b-smoR & $\begin{array}{l}\text { IPTG inducible expression vector carrying } \\
\text { smoR ORF, } A m p^{r}\end{array}$ & This work \\
\hline $\begin{array}{l}\text { pBBR1MCS-5- } \\
\text { lacZ }\end{array}$ & $\begin{array}{l}\text { pBBR1MCS-5 plasmid carrying } \\
\text { promoterless lacZ gene, } \mathrm{Gm}^{r}\end{array}$ & $\begin{array}{l}\text { Fried et al., } \\
2012\end{array}$ \\
\hline $\begin{array}{l}\text { pBBR1MCS-5- } \\
\text { PsmoR::lacZ }\end{array}$ & $\begin{array}{l}\text { pBBR1MCS-5 plasmid carrying fusion } \\
\text { PsmoR::lacZ gene, } \mathrm{Gm}^{r}\end{array}$ & This work \\
\hline pME6000 & Broad-host-range cloning vector, $T^{r}$ & $\begin{array}{l}\text { Maurhofer } \\
\text { et al., } 1998\end{array}$ \\
\hline pMEPlac::aiiA & $\begin{array}{l}\text { pME6000 carrying lactonase aiiA gene } \\
\text { from } B \text {. subtilis under the control of Plac } \\
\text { promoter, } T C^{r}\end{array}$ & $\begin{array}{l}\text { Reimmann } \\
\text { et al., } 2002\end{array}$ \\
\hline
\end{tabular}

electroporated (Choi et al., 2006) into S. maltophilia E77 and transformants were seeded onto LB plates supplemented with $40 \mu \mathrm{g} / \mathrm{ml} \mathrm{Gm}$.

To generate the expression vector for SmoR production in E. coli, the ORF of smlt1839 was amplified using primer pair P1ExpSmoR-P2ExpSmoR and the amplified fragment was digested with $\mathrm{NdeI}$ and $\mathrm{XhoI}$ and cloned into their respective restriction sites into pET22b (Novagen), creating pET22b-smoR. E. coli strain BL21 (DE3) was transformed (Sambrook et al., 1989) with plasmid pET22b-smoR and transformants were seeded onto LB plates containing $20 \mu \mathrm{g} / \mathrm{ml} \mathrm{Amp.}$

The vectors pME6000 (Maurhofer et al., 1998) and pMElacZ::aiiA (Reimmann et al., 2002) - the latter carrying a transcriptional fusion between lacZ promoter and the ORF of the lactonase AiiA from Bacillus subtilis strain A24 (Dong et al., 2000)-were provided by the authors and were used to investigate the effect of the lactonase AiiA on the degradation of the AHL signals from $P$. aeruginosa. Both vectors were electroporated (Choi et al., 2006) into P. aeruginosa MPAO1 and transformants were seeded onto LB plates containing $17 \mu \mathrm{g} / \mathrm{ml} \mathrm{Tc}$.

\section{Generation of $\Delta s m o R$ Mutant}

S. maltophilia E77 $\Delta s m o R$ mutant was obtained by allelicexchange recombination using erythromycin as antibioticresistance cassette. Briefly, $s m o R$ upstream and downstream flanking regions (993 and $863 \mathrm{bp}$, respectively) were amplified by PCR using primer pairs P1MutSmoR-P2MutSmoR (upstream region) and $\mathrm{P} 3 \mathrm{MutSmoR}-\mathrm{P} 4 \mathrm{MutSmoR}$ (downstream region) and inserted, flanking an erythromycin cassette, into the suicide vector pEX18Tc (Hoang et al., 1998), generating plasmid pEXsmoR. The erythromycin cassette was previously amplified from plasmid pGEM-Erm (Table 2) using primers PErm5' and 
TABLE 3 | Strains used in this study.

\begin{tabular}{|c|c|c|}
\hline Strains & Relevant characteristics & References \\
\hline \multicolumn{3}{|l|}{ S. maltophilia } \\
\hline E77 & Wild type & $\begin{array}{l}\text { Ferrer- } \\
\text { Navarro et al. } \\
2013\end{array}$ \\
\hline $\mathrm{E} 77 \Delta s m o R$ & E77 $\Delta s m o R(\Delta s m l t 1839), \mathrm{Erm}^{r}$ & This work \\
\hline $\begin{array}{l}\text { E77 } \\
\text { pBBR1MCS-5-lacZ }\end{array}$ & $\begin{array}{l}\text { E77 harboring vector } \\
\text { pBBR1MCS-5-lacZ, Gm }\end{array}$ & This work \\
\hline $\begin{array}{l}\text { E77 pBBR1MCS-5- } \\
\text { PsmoR::lacZ }\end{array}$ & $\begin{array}{l}\text { E77 harboring vector } \\
\text { pBBR1MCS-5-PsmoR::/acZ, Gmr }\end{array}$ & This work \\
\hline $\begin{array}{l}\text { E77 } \Delta s m o R \\
\text { pBBR1MCS-5-lacZ }\end{array}$ & $\begin{array}{l}\text { E77 } \triangle s m o R \text { harboring vector } \\
\text { pBBR1MCS-5-lacZ, Gmr, Erm }\end{array}$ & This work \\
\hline $\begin{array}{l}\text { E77 } \triangle \text { smoR } \\
\text { pBBR1MCS-5- } \\
\text { PsmoR::lacZ }\end{array}$ & $\begin{array}{l}\text { E77 } \Delta s m o R \text { harboring vector } \\
\text { pBBR1MCS-5-PsmoR::lacZ, Gm }{ }^{r} \text {, } \\
\text { Erm }^{r}\end{array}$ & This work \\
\hline \multicolumn{3}{|l|}{ E. coli } \\
\hline $\mathrm{DH} 5 \alpha$ & 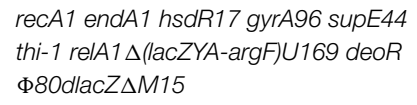 & $\begin{array}{l}\text { Lab. } \\
\text { Collection }\end{array}$ \\
\hline $\mathrm{DH} 5 \alpha \mathrm{pEXsmoR}$ & $\begin{array}{l}\mathrm{DH} 5 \alpha \text { harboring vector } \mathrm{pEX} s m o R \text {, } \\
\mathrm{TC}^{r}, \mathrm{Erm}^{r}\end{array}$ & This work \\
\hline BL21 (DE3) & $\begin{array}{l}\text { fhuA2 [lon] ompT gal ( } \lambda \text { DE3) [dcm] } \\
\Delta h s d S \lambda \text { DE3 }=\lambda \text { sBamHlo } \\
\Delta E c o R l-B \text { int::(lacl::PlacUV5::T7 } \\
\text { gene1) i21 } \Delta \text { nin5 }\end{array}$ & Novagen \\
\hline BL21 (DE3) pET22b & BL21 (DE3) harboring pET22b, Amp ${ }^{r}$ & This work \\
\hline $\begin{array}{l}\text { BL21 (DE3) } \\
\text { pET22b-smoR }\end{array}$ & $\begin{array}{l}\text { BL21 (DE3) harboring pET22b-smoR, } \\
\text { Amp }^{r}\end{array}$ & This work \\
\hline \multicolumn{3}{|l|}{$P$. aeruginosa } \\
\hline MPAO1 & Wild type & $\begin{array}{l}\text { Jacobs et al., } \\
2003\end{array}$ \\
\hline MPAO1 pME600 & MPAO1 harboring pME600, $\mathrm{Tc}^{r}$ & \\
\hline \multicolumn{3}{|l|}{ A. tumefaciens } \\
\hline KYC55 & $\begin{array}{l}\text { KYC55 harboring vectors pJZ384, } \\
\text { pJZ410 and pJZ372 Spc }{ }^{r}, \mathrm{Gm}^{r}, \mathrm{Tc}^{r}\end{array}$ & $\begin{array}{l}\text { Zhu et al., } \\
2003\end{array}$ \\
\hline
\end{tabular}

PErm3'. S. maltophilia E77 was electroporated (Choi et al., 2006) with the suicide vector pEXsmoR and transformants were seeded onto LB plates containing $500 \mu \mathrm{g} / \mathrm{mL}$ Erm and subsequently streaked onto LB plates containing $17 \mu \mathrm{g} / \mathrm{mL}$ Tc to discard single cross-over events. $s m o R$ deletion was also verified by PCR using primer combinations P1DemSmoR-PErm5'rev (for upstream region) and P2DemSmoR-PErm $3^{\prime}$ rev (for downstream region). The obtained fragments were subsequently verified by sequencing (Macrogen).

\section{Measuring B-Galactosidase Activity}

To evaluate the expression levels of hchA-smoR promoter, Bgalactosidase assays were performed for the strains E77 wild type and $\triangle s m o R$ mutant harboring either the vectors pBBR1MCS-5PsmoR:lacZ or pBBR1MCS-5-lacZ-the latter used as a controlduring growth in $\mathrm{LB}$ medium at $30^{\circ} \mathrm{C}$, following the protocol described by Miller (1972). All bacterial cultures were started with an initial inoculum corresponding to an optical density at $550 \mathrm{~nm}\left(\mathrm{OD}_{550}\right)$ of 0.05 . To determine the activity of the $h c h A$-smoR promoter during growth curve, $0.1 \mathrm{ml}$-samples were taken at different times from 4 to $48 \mathrm{~h}$. To investigate the effect of the presence of AHL molecules in the activity of the $h c h A$-smoR promoter, initial cultures were supplemented with various synthetic AHLs (Cayman Chemical) -C6-HSL, oxo-C8HSL, C8-HSL, and C10-HSL- with different concentrations (1 up to $10 \mu \mathrm{M}$ ), and $0.1 \mathrm{ml}$ samples were taken and measured after $24 \mathrm{~h}$ and $48 \mathrm{~h}$ of incubation at $30^{\circ} \mathrm{C}$. After analyzing the data we determined $\beta$-galactosidase specific activities in Miller Units (Miller, 1972). All AHL stocks were solubilized in $70 \%$ acetonitrile/water acidified with $0.1 \mathrm{M} \mathrm{HCl}$ final concentration. All experiments were performed by triplicate and comparison of $ß$-galactosidae activity was performed by One-Way analysis of variance (ANOVA) with a Bonferroni's multiple comparison post-test.

\section{Extraction, Thin Layer Chromatography and Bioassay of AHLs}

To evaluate AHL produced by $P$. aeruginosa, $150 \mathrm{ml}$ culture supernatants of strain MPAO1, or MPAO1 transformed with either pME6000 or pMElacZ::aiiA grown in LB at $37^{\circ} \mathrm{C}$ for $24 \mathrm{~h}$ $\left(\mathrm{OD}_{550}\right.$ of about 2$)$, were extracted with $300 \mathrm{ml}$ of acidified ethyl acetate $(0.1 \%$ acetic acid). The organic phase was evaporated to dryness using a rotary evaporator, and the residues were dissolved in an appropriate volume of acidified ethyl acetate. $5 \mu \mathrm{l}$ aliquots of dissolved ethyl acetate residues were spotted onto C18 reverse-phase plate (Merck) (Shaw et al., 1997) and separated with methanol:water $(60: 40, \mathrm{vol} / \mathrm{vol})$ as running solvent. TLC plates were subsequently air-dried for at least $1 \mathrm{~h}$ and overlaid with $100 \mathrm{ml}$ of unsolidified warm AT medium containing $0.8 \%$ agar, $60 \mu \mathrm{g} / \mathrm{ml} \mathrm{X-Gal} \mathrm{and} \mathrm{the} \mathrm{AHL} \mathrm{reporter}$ strain KYC55 to an $\mathrm{OD}_{550}$ of ca. 0.8. TLC plates were incubated overnight at $30^{\circ} \mathrm{C}$, and AHL activity was identified by the presence of blue spots. $2 \mu \mathrm{l}$ of the aforementioned synthetic AHLs were also tested in TLC coupled to bioassay and used as a control.

\section{AHL Binding Assay}

The AHL binding assay was performed as described (Subramoni and Venturi, 2009b), with few modifications. $20 \mathrm{ml}$ cultures of $E$. coli BL21 (DE3) harboring either pET22b or pET22b-smoR were grown at $37^{\circ} \mathrm{C}$ in $\mathrm{LB}$ medium containing $10 \mu \mathrm{g} / \mathrm{ml} \mathrm{Amp} \mathrm{to} \mathrm{an}$ $\mathrm{OD}_{550}$ of 0.1 . Bacterial cultures were then supplemented with different AHL molecules (C6-HSL, oxo-C8-HSL, C8-HSL and C10-HSL) at 10 and $20 \mu \mathrm{M}$ final concentration and cultures were incubated until reaching an $\mathrm{OD}_{550}$ of 0.6 . SmoR production was induced with $1 \mu \mathrm{M}$ final concentration of IPTG and the cultures were additionally incubated for $3.5 \mathrm{~h}$. $\mathrm{OD}_{550}$ was measured and the cultures were adjusted to contain an equal number of cells per $\mathrm{mL}$ and subsequently centrifuged. Cell pellets were washed three times with $10 \mathrm{ml}$ of PBS and cellular suspensions were extracted twice with the same volume of acidified ethyl acetate. The extracts were then dried, dissolved in ethyl acetate and analyzed by TLC coupled to AHL bioassay, as described above. An aliquot of the corresponding induced culture was previously removed to control the identity of the overproduced recombinant protein (see Supplementary Figure S1). 


\section{Swarming Assay}

Swarming motility was assayed on BM2 medium plates $(62 \mathrm{mM}$ potassium phosphate buffer, $\mathrm{pH}=7,2 \mathrm{mM} \mathrm{MgSO}_{4}, 10 \mu \mathrm{M}$ $\mathrm{FeSO}_{4}, 0.5 \%$ [wt/vol] casamino acids, supplemented with glucose $0.4 \%$ and solidified with $0.5 \%$ BD Difco Noble agar) (Overhage et al., 2007). Plates containing $20 \mathrm{ml}$ of fresh swarm medium were dried under a laminar-flow hood for $20 \mathrm{~min}$ before pin-inoculation. When indicated, solidified swarm plates were supplemented with $10 \mu \mathrm{l}$ of concentrated culture supernatantextracted as described above- of $P$. aeruginosa MPAO1 and its derivative strains, as indicated in figure captions. Inoculated swarm plates were sealed to maintain the humidity and incubated at $30^{\circ} \mathrm{C}$ up to five days. Swarming experiments were done in triplicate and representative images are shown.

\section{Results}

\section{Smlt1839 Contains both the AHL- and DNA-Binding LuxR Domains}

It is known that certain non AHL-producing bacteria are able to sense AHLs and regulate various biological functions in response to signals produced by others through diverse LuxRlike regulators (Patankar and González, 2009). The genome of S. maltophilia strain K279a (Crossman et al., 2008) was revisited for the presence of genes encoding putative LuxRlike regulators. Besides the eight genes already annotated as two-component-system response regulators of the LuxR family, a total of seven additional hypothetical LuxR regulators were identified (Table 4), none of them associated to a luxI homolog. All these LuxR-solo candidates were examined in detail for the presence of the typical N-terminal AHL-binding domain (PFAM 03472) and the C-terminal helix-turn-helix (HTH) DNA-binding domain (PFAM 00196) (Miller and Bassler, 2001). From these, only the gene smlt1839 was found to encode for a protein-here named SmoR ( tenotrophomonas maltophilia orphan regulator)-containing both conserved domains (Table 4). A subsequent protein alignment with known orphan regulators from distinct Proteobacteria including PpoR from $P$. putida, SdiA from S. enterica, OryR from $X$. oryzae pv. oryzae, and TraR from A. tumefaciens, revealed that at the N-terminal domain four out of six residues involved in AHL binding (Patankar and González, 2009) are conserved in SmoR (Figure 1). Concerning the C-terminal HTH domain, the three residues responsible for DNA binding (Hanzelka and Greenberg, 1995; Fuqua et al., 1996) are also conserved in SmoR (Figure 1). Further protein BLAST analysis revealed that SmoR is largely conserved among S. maltophilia (data not shown). These results suggest that the conserved regulator SmoR (Smlt1839) could be implicated in signaling systems in $S$. maltophilia.

\section{S. maltophilia SmoR Binds AHLs}

It has been demonstrated that various LuxR solos containing the AHL-binding domain are able to bind to one or more AHL signal molecules. To determine whether in S. maltophilia the regulator SmoR could bind to any of these signals, an AHLbinding assay was performed. The appropriate overexpression of S. maltophilia smoR in E. coli strain BL21 (DE3) was validated by MALDI-MS analysis prior to initiate the AHL-binding assay (see Supplementary Figure S1).

E. coli BL21 (DE3) harboring either the empty vector pET$22 \mathrm{~b}$ or the one overproducing SmoR were grown in a rich medium supplemented with a variety of AHLs (see Materials and Methods). After the appropriate incubation time, the culture supernatant was removed and the cell pellet was washed and subsequently extracted with acidified ethyl acetate. Concentrated cell extracts were visualized by TLC coupled to the AHL bioassay, resulting in the detection of the signal oxo-C8-HSL (Figure 2). Likewise, it was observed that the detection depends on AHL concentration, since the culture supplemented with $20 \mu \mathrm{M}$ oxo-C8-HSL presented a more intense spot compared to that

TABLE 4 | Hypothetical LuxR-like regulators annotated in the genome of S. maltophilia strain K279a.

\begin{tabular}{|c|c|c|c|c|}
\hline Locus ID & Lenght & N-ter Domain & C-ter Domain & Annotation in K279a \\
\hline Smlt1839 & 234 & $\mathrm{AHL}$ & LuxR HTH & LuxR family transcriptional regulator \\
\hline Smlt0195 & 212 & REC & LuxR HTH & LuxR family two component response regulator \\
\hline Smlt0389 & 223 & REC & LuxR HTH & Two component transcriptional regulator, LuxR family \\
\hline Smlt2299 & 210 & REC & LuxR HTH & Response regulator protein LuxR family \\
\hline Smlt2366 & 208 & REC & LuxR HTH & Two-component response regulator, LuxR family \\
\hline Smlt4224 & 212 & REC & LuxR HTH & LuxR family two-component response regulator \\
\hline Smlt0367 & 200 & REC & LuxR HTH & Two-component system response regulator, LuxR family \\
\hline Smlt0400 & 254 & REC & LuxR HTH & Two-component response regulator transcriptional regulator \\
\hline Smlt0881 & 213 & REC & LuxR HTH & Two-component response regulator transcriptional regulator \\
\hline Smlt1255 & 213 & REC & LuxR HTH & Two-component response regulator transcriptional regulator \\
\hline Smlt1788 & 215 & REC & LuxR HTH & Two-component response regulator transcriptional regulator \\
\hline Smlt2595 & 224 & REC & LuxR HTH & Two-component response regulator transcriptional regulator \\
\hline Smlt2658 & 213 & REC & LuxR HTH & Two-component response regulator transcriptional regulator \\
\hline Smlt2891 & 217 & REC & LuxR HTH & Two-component response regulator transcriptional regulator \\
\hline Smlt4624 & 221 & REC & LuxR HTH & Two component system response regulator \\
\hline
\end{tabular}




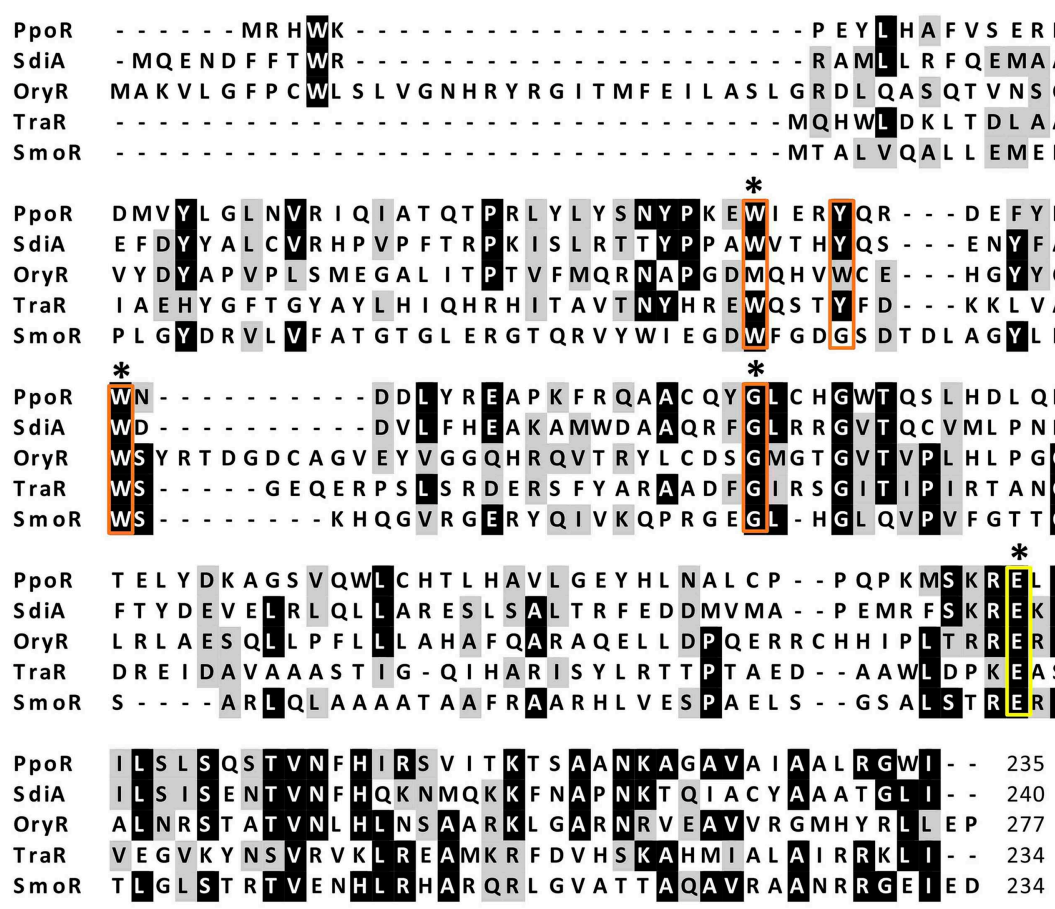

FIGURE 1| Protein alignment of orthologs LuxR solos from diverse Proteobacteria; PpoR (FM992078): Pseudomonas putida strain RD8MR3; SdiA (AAC08299.1): Salmonella enterica subsp. enterica serovar Typhimurium strain LT2; OryR (AAR91700.1): Xanthomonas oryzae pv. oryzae; TraR
(AAZ50597.1): A. tumefaciens; SmoR: S. maltophilia E77 (KP691985). Red boxes highlight amino acids implicated in $A H L$ binding and yellow boxes indicate residues involved in DNA binding (HTH). From these, conserved amino acids in E77 are marked with an asterisk. supplemented with $10 \mu \mathrm{M}$. On the other hand, cell extracts from E. coli cells containing the empty vector did not show detectable AHL activity (Figure 2). The experiment was performed by triplicate and non-systematic binding was observed for the other AHLs tested. Overall, this indicates that $S$. maltophilia could sense AHL signal molecules -in particular oxo-C8-HSL- through the LuxR solo SmoR.

\section{hchA and smoR Are Part of the Same Operon, and Operon Expression is Growth-phase- and AHL-Dependent}

In S. maltophilia K279a, the gene encoding the regulator SmoR is localized downstream of the gene encoding for the chaperone HchA (Smlt1840), separated by only five nucleotides, indicating that both genes could form the operon hchA-smoR. This genetic organization is conserved in all available $S$. maltophilia genomes and also in the clinical strain E77 used in the present study. The upstream genes to $h c h A$ in strains D457 and JV3 are oriented in the opposite direction, indicating that there must be a promoter preceding $h c h A$ and confirming the existence of a bicistronic operon. Interestingly, this operon has been observed only in few Gammaproteobacteria, including Pseudomonas spp., Vibrio spp., Acinetobacter spp., Serratia spp., among few others, as predicted by InterPro (Family IPR019941). Accordingly, these regulator elements are annotated as "LuxR chaperone HchA-associated". In E. coli, the gene hchA encodes for the chaperone Hsp31, which

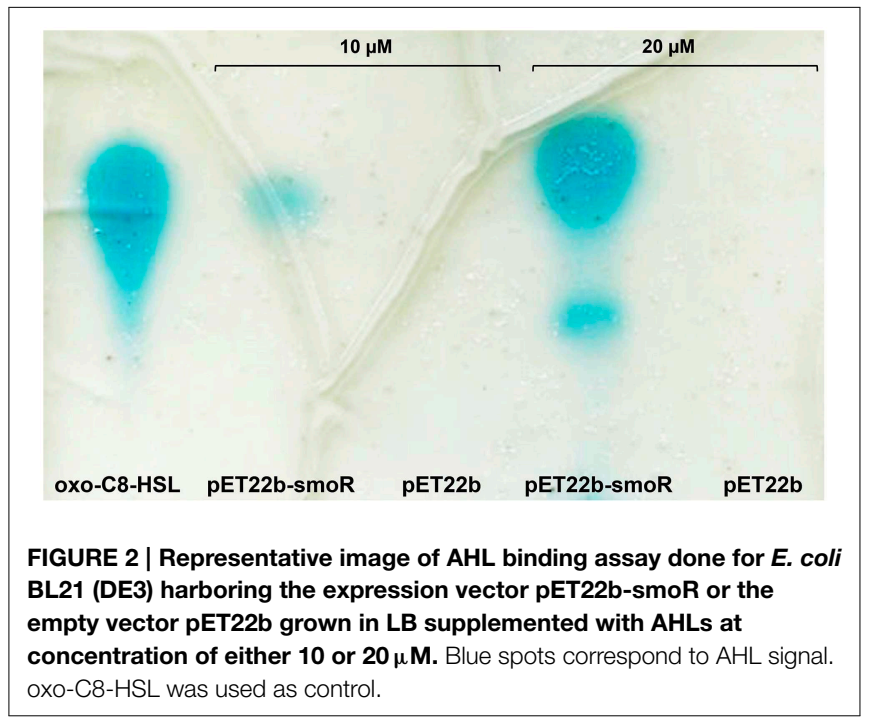

displays a high protein identity (60\%) to S. maltophilia HchA. However, E. coli hchA is an isolated gene in this bacterial genome. It has been shown that Hsp31 is a glyoxalase that plays a central role in detoxification of dicarbonyl radicals (Subedi et al., 2011) as well as in the response to bacterial stress such as heat shock (Mujacic et al., 2004; Mujacic and Baneyx, 2006) and acidic 
conditions (Mujacic and Baneyx, 2007). In these mentioned studies it has been reported that transcription of $h c h A$ is induced at high population density.

In S. maltophilia E77 the upstream hchA-smoR operon region was examined for the presence of a putative promoter. Despite canonical core promoter elements are not found in this region, DNA sequence alignment of this region from different $S$. maltophilia strains revealed a conserved ribosomebinding site and a palindromic conserved motif spanning from positions -75 to -56 with respect to the translational start site (Figure 3A). The genome of S. maltophilia K279a was also evaluated for the presence of this palindromic motif in other promoter gene regions. Curiously, only a highly similar box (68.75\% identity) was found in the intergenic region between smlt2137 (position -143 to -159 ) and smlt2138 (position -15 to -31 ). Those genes encode for a universal stress-family protein (Smlt2137) and for the transcriptional regulator NfxB (Smlt2138) (Shiba et al., 1995), respectively.

The hchA-smoR upstream region including the palindromic sequence motif was fused to lac $Z$ gene and used in $B$ galactosidase experiments to corroborate the existence of a promoter. The expression levels of the fusion construct PsmoR::lacZ were monitored in S. maltophilia strain E77 wild type and its derivative $\triangle s m o R$ mutant, in order to determine whether the expression pattern of the operon was similar to that previously described for the $h c h A$ gene in E. coli (Mujacic and Baneyx, 2006, 2007). Additionally, the role of SmoR in such expression was also evaluated, since the autoregulation of LuxR proteins is relatively common (Shadel and Baldwin, 1992; Chatterjee et al., 1996; Minogue et al., 2002).

The results from the $B$-galactosidase experiments indicate that the promoter activity is slight at low cell densities and increases with bacterial-growth rate, showing a maximum at $48 \mathrm{~h}$ (stationary phase, Figure 3B). Although this tendency was observed in both wild type and $\Delta s m o R$ backgrounds, the absence of SmoR led to increased levels of expression compared to the wild type strain E77, specially at late stationary phase of growth (Figure 3B) $(P<0.01)$. Under these standard conditions (LB and $30^{\circ} \mathrm{C}$ ) all tested strains showed similar growth curves reaching stationary phase by $24 \mathrm{~h}$ approximately. These results suggest that operon components would act at high cell densities, participating likely in this sort of stress response as observed for Hsp31 in E. coli.

Since we observed in vitro that SmoR bind the signal oxoC8-HSL (Figure 2) and it is known that some active LuxRlike proteins can autoregulate their own expression, we wanted to investigate the role of SmoR on the regulation of $h c h A-$ $s m o R$ operon expression in the presence of AHLs. To do that, $\beta$-galactosidase assays were performed for the same strains supplemented with various AHLs (see Materials and Methods).

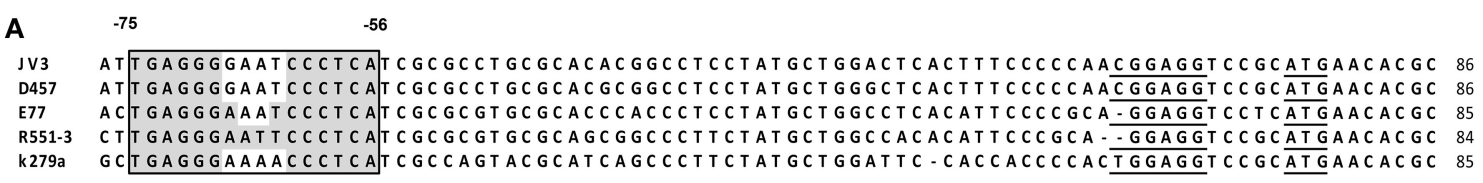

B

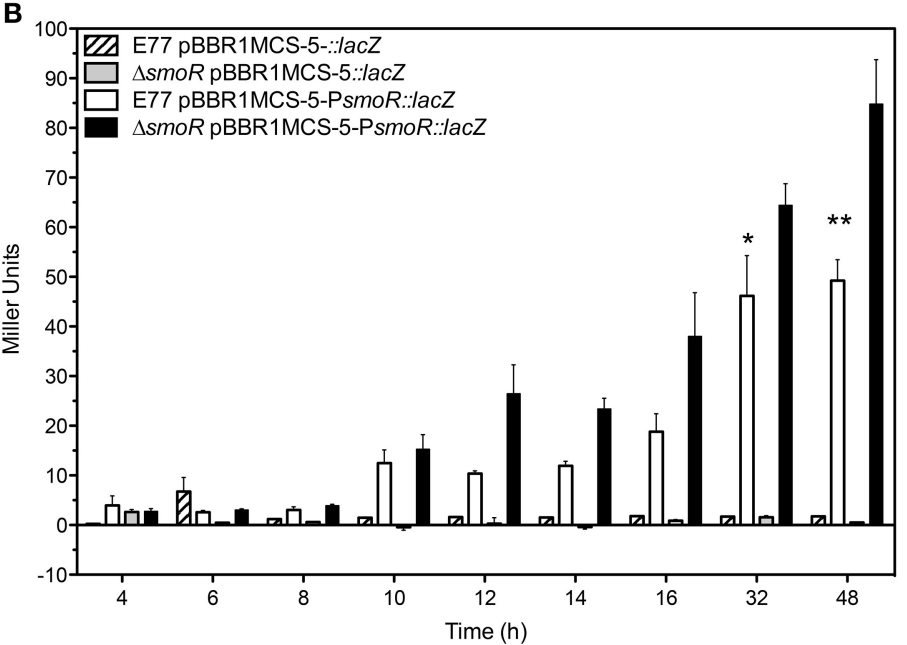

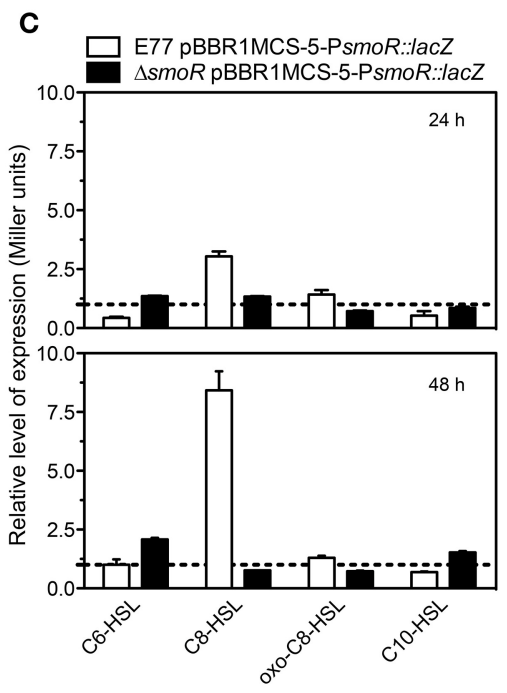

FIGURE 3 | (A) Alignment of the hchA-smoR promoter from different $S$. maltophilia strains. Gray shadow indicates the palindromic motif. Underlined sequences correspond to the putative ribosome binding site (RBS) and the start codon (ATG). (B) B-galactosidase assay expressed in Miller Units of E77 WT and $\triangle S m o R$ mutant both harboring either pBBR1MCS-5::/acZ or pBBR1MCS-5-PsmoR::/acZ monitored during 48h. Under these conditions all tested strains showed similar growth curves.
(C) B-galactosidase activity of E77 wild type and $\triangle s m o R$ mutant both harboring vector pBBR1MCS-5-PSmoR::/acZ grown in LB supplemented with diverse AHLs at $1 \mu \mathrm{M}$ concentration each and incubated at $30^{\circ} \mathrm{C}$ for 24 or $48 \mathrm{~h}$. Relative expression values are reported as Miller units of $\beta$-galactosidase activity in cells grown under the indicated growth conditions divided by the values in cells grown in LB broth without AHLs. ${ }^{\star} P<0.05 ;{ }^{* *} p<0.01$. 
The results showed that the expression of the operon was also modulated by the presence of these signal molecules. In particular, supplementation of E77 wild type with $1 \mu \mathrm{M} \mathrm{C} 8$ HSL resulted into approximately threefold and 8 -fold operon activation at 24 and $48 \mathrm{~h}$ post induction respectively, compared to the expression levels of the supplemented $\Delta s m o R$ mutant (Figure 3C). This indicates that SmoR is involved in AHLdependent operon-expression regulation.

\section{AHLs Produced by $P$. aeruginosa Promote Swarming Motility in S. maltophilia, SmoR Playing a Central Role in this Stimulation}

It is known that AHL signals modulate several biological functions not only in AHL-producing bacteria, but also in certain species lacking typical luxI/luxR systems which are still able to respond to exogenous AHLs through diverse orphan LuxR. One of the behaviors that have raised more interest recently and is commonly regulated by AHL-QS is swarming motility (Daniels et al., 2004). Swarming motility is a rapid and coordinated translocation of a bacterial population across semi-solid surfaces, which frequently requires quorum sensingmediated synchronization (Kearns, 2010).

Since S. maltophilia frequently cohabit with AHL-producing bacteria -i.e., P. aeruginosa (Moskowitz et al., 2005)- we investigated the effect that $P$. aeruginosa AHLs could have on $S$. maltophilia regulation, more specifically on swarming motility. To that end, we supplemented S. maltophilia swarming plates with concentrated supernatants of $P$. aeruginosa strain MPAO1 wild type and MPAO1 harboring the plasmid pMEPlac::aiiA, which expresses the lactonase AiiA from B. subtilis (Dong et al., 2000). In order to corroborate the effect of the lactonase AiiA in AHL degradation, TLC followed by AHL bioassay was performed in parallel to swarming assays. As shown in Figure 4, expression of AiiA led to a decrease of $P$. aeruginosa AHL production and, interestingly, it resulted in a drastic reduction of S. maltophilia swarming stimulation. These results would indicate that AHL signals produced by $P$. aeruginosa promote swarming motility in S. maltophilia.

Several evidences drive us to suggest that SmoR was responsible for the observed AHL-mediated swarming stimulation in S. maltophilia strain E77. It has been shown that, in the related bacteria $X$. oryzae pv. oryzae, the LuxR solo OryR also regulates certain motility processes, including swarming (González et al., 2013). In order to test such hypothesis in S. maltophilia, MPAO1 concentrated supernatants were spotted onto swarming plates seeded with the strains E77 wild type and the $\triangle s m o R$ mutant. The results further demonstrate that, as observed before, the MPAO1 concentrated supernatant significantly promotes the swarming ability of E77 (Figure 5). To the contrary, mutation of $s m o R$ resulted into a loss of swarming motility and supplementation with $P$. aeruginosa concentrated supernatant produced only a minor stimulation compared to E77 WT, perhaps due to the surfactant character of the AHL signals and other hydrophobic molecules present in its supernatant. These results strongly suggest that AHLs produced exogenously promote swarming motility in S. maltophilia and SmoR plays a central role in such stimulation (Figure 5).

\section{Discussion}

The interest in LuxR-solo investigation has grown among microbiologists, since these regulatory elements may unveil novel signaling systems. The increment of public sequenced genomes has permitted scientists to screen an extraordinary number of bacterial genomes and identify new regulator elements. It recently has been shown in bacteria that $75 \%$ of the annotated luxR-type genes encode for a "LuxR solo" (Hudaiberdiev et al., 2015), which is a surprisingly high proportion. To date, it is known that various LuxR can regulate a range of biological function in response to: endogenous AHLs (Chugani et al., 2001; Lequette et al., 2006); exogenous AHLs produced by neighboring bacteria (Ahmer et al., 1998; Michael et al., 2001; Ahmer, 2004; Yao et al., 2006); the novel bacterial signaling molecules dialkylresorcinols (DARs) and cyclohexanediones (CHDs) (Brameyer et al., 2015); or even signals produced by plants (Ferluga et al., 2007; Ferluga and Venturi, 2009). Considering the high heterogeneity and complexity of LuxR solos in bacteria, it is highly probable that new regulatory networks are there to discover.

The aim of the present study was to identify and investigate putative LuxR solos in S. maltophilia. After screening the genome of the model strain K279a (Crossman et al., 2008), only the protein encoded by the smlt1839 gene (SmoR) was found to display the two typical LuxR domains: the autoinducerbinding domain (Shadel et al., 1990; Slock et al., 1990) and the DNA-binding helix-turn-helix (HTH) domain (Choi and Greenberg, 1991; Fuqua and Winans, 1994). Analysis at the amino-acid sequence level revealed that from the 9 crucial residues (Whitehead et al., 2001; Zhang et al., 2002), seven are conserved in SmoR. Diverse structural and functional analyses of several LuxR regulators have revealed that, while the DNAbinding domain (HTH) is widely conserved, the autoinducerbinding domain presents substantial variability, likely to adjust to a range of signal molecules (Vannini et al., 2002; Zhang et al., 2002; Yao et al., 2006; Bottomley et al., 2007). This is the situation observed in S. maltophilia SmoR, where the AHL-binding domain presents substitutions in residues 62 and 74, while the HTH domain is perfectly conserved (Figure 1). It has been reported that in A. tumefaciens, the LuxR solo TraR also binds to oxo-C8-HSL through a hydrophobic cavity composed of six conserved residues (Vannini et al., 2002; Zhang et al., 2002). Single-mutation analysis of some of these residues does not render AHL-binding incompetent TraR, but elevated signal concentrations -5000 to 10,000 -fold- are then needed to achieve oxo-C8-HSL binding (Koch et al., 2005).

Additionally, it has been demonstrated that in $X$. oryzae pv. oryzae (Xoo), the LuxR solo OryR-which presents different critical amino acids in the AHL-binding domain- recognizes plant signals rather than AHLs (Ferluga et al., 2007; Ferluga and Venturi, 2009; González et al., 2013). Taking into account this observations and considering the variation in the AHL-binding domain of SmoR, it has been suggested that S. maltophilia SmoR might also recognize plant signals (Crossman et al., 2008), a possibility that has not been yet validated. We show here 


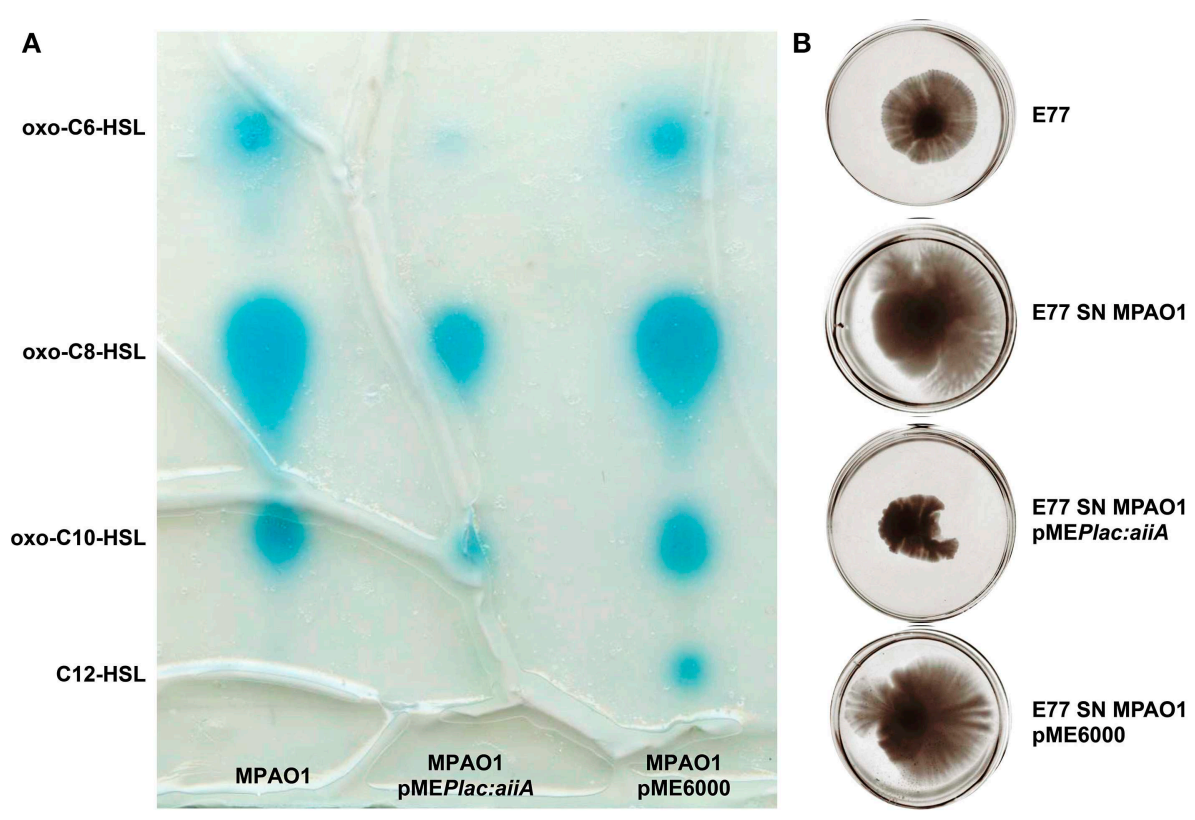

FIGURE 4 | Representative images of (A) TLC coupled to AHL bioassay of concentrated supernatants of $P$. aeruginosa MPAO1, MPAO1 pMEPlac::aiiiA, and MPAO1 pME6000. Blue spots correspond to
AHL molecules. (B) Swarming motility in BM2 0.5\% agar medium plates of S. maltophilia E77 supplemented with $10 \mu \mathrm{l}$ of the same concentrated supernatants $(\mathrm{SN})$ and incubated at $30^{\circ} \mathrm{C}$ for 5 days.

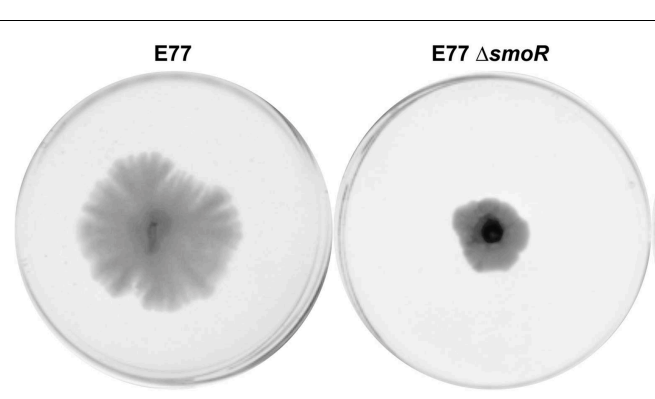

FIGURE 5 | Representative images of swarming motility in BM2 0.5\% agar medium plates of S. maltophilia E77 WT and $\Delta s m o R$ mutant supplemented with $10 \mu$ I of concentrated supernatants (SN) of $P$.

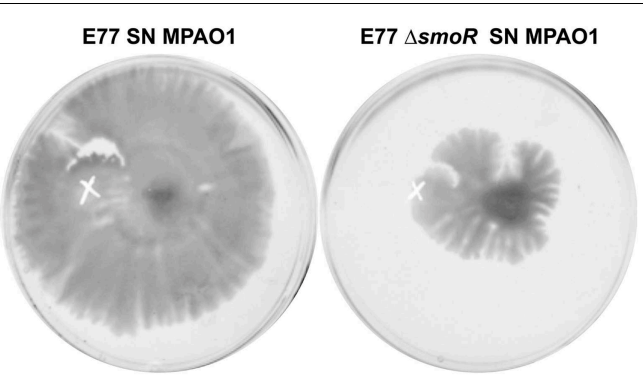

aeruginosa strain MPAO1 WT and incubated at $30^{\circ} \mathbf{C}$ for 5 days. The white cross indicates the zone of the addition of the concentrated supernatants. that SmoR binds AHLs, in particular the signal oxo-C8-HSL (Figure 2).

Genomic organization and sequence analysis have shown that oryR and $x c c R$ are not orthologous to $s m o R$. Indeed, SmoR belongs to a recently classified subfamily designated "LuxRlike regulators chaperone $\mathrm{HchA}$ associated" as predicted by InterPro (http://www.ebi.ac.uk/interpro/) (Mitchell et al., 2014). Curiously, most bacteria sharing this particular operon display AHL production. However, there has been no functional study on the hchA-smoR operon. Nevertheless, the high homology observed between S. maltophilia HchA and E. coli Hsp31 suggests that, apart from being orthologous genes, both HchA and Hsp31 may regulate similar functions. In the genome of $E$. coli, the $h c h A$ gene is monocistronic. Its gene product Hsp31 has been widely studied in E. coli and its diverse functions have been elucidated. Initially, Hsp31 was reported to be a heat-inducible chaperone, showing feeble protease activity (Sastry et al., 2002; Malki et al., 2003, 2005). Further, it was shown that Hsp31 participates in heat shock and starvation response (Mujacic et al., 2004; Mujacic and Baneyx, 2006) as well as resistance to acid environments, generated during the stationary phase of growth (Mujacic and Baneyx, 2007). Recently it has been demonstrated that Hsp31 is a glyoxalase that participates in the detoxification of dicarbonyl stress (Subedi et al., 2011), which spans even more the functional spectrum of this versatile protein. Overall, this protein appears to act in front of stress environments derived from high cell density, a situation where QS systems are also active. In this line, we have shown that in S. maltophilia also a high cell density induces the expression of the hchA-smoR operon (Figure 3B). This suggests that S. maltophilia HchA may also act in front of stress situations such as starvation or accumulation of secondary metabolites, as described for E. coli 
Hsp31 (Mujacic and Baneyx, 2006, 2007; Subedi et al., 2011). In addition, we have observed that mutation of $s m o R$ results in a higher activity of operon during growth, which suggests that SmoR may act as a repressor in this situation (Figure 3B). On the other hand, we have observed in the wild type background that the presence of the exogenous signal C8-HSL significantly enhance in vivo promoter activity (Figure 3C). Although no systematic binding was observed for C8-HSL in the AHL-binding assay (data not shown), ß-galactosidase experiments make us reconsider whether SmoR could also bind to this signal, apart from oxo-C8-HSL. A possible explanation could rely on the use of different systems in each technique (note that AHL-binding assay was done in E. coli, while ß-galactosidase experiments have been performed in S. maltophilia). Therefore, further studies will be necessary to better understand the role of both eightcarbon AHL in the SmoR-dependent regulation. Interestingly, we have observed that the LuxR solos QscR (PA1898) from $P$. aeruginosa and the SmoR are putative orthologs. A new in vivo mechanism for LuxR activation depending on the free and signalbond state has been proposed for QscR (Oinuma and Greenberg, 2011). It has been reported that although QscR binding to the signal 3-oxo-C6-HSL has a slightly effect on its own transcription (Lee et al., 2006), it can stimulate dimerization and activation, while preventing it from proteolysis (Oinuma and Greenberg, 2011; Chugani and Greenberg, 2014). A similar mechanism have been described for TraR regulator, suggesting that this new mechanisms might be widespread among LuxR homologs (Zhu and Winans, 1999, 2001), perhaps including SmoR. Altogether, we hypothesize that the regulator $\mathrm{SmoR}$ might bind to the palindromic box and block operon expression, until the presence of signal molecules bind to $\mathrm{SmoR}$ and avoid operon repression. Curiously, a highly related palindromic box was also identified between genes smlt2137 (universal stress family protein) and smlt2138 (transcriptional regulator $n f x B$ ) (Mitchell et al., 2014), two proteins that appear to be also involved in stress response. Nevertheless, determining whether SmoR could play an activator or a repressor role as well as which other genes could be under its regulation is something that will require further studies. Overall, our results suggest that in S. maltophilia, the hchA-smoR operon could have two important functions: (i) detoxification and recycling of secondary metabolites (HchA) and (ii) response to QS signals (SmoR), both deriving from situations of high cell density.

Interactions within microbial populations are common and essential for community maintenance (Ryan and Dow, 2008). In vitro studies of microbial consortia represent a first approach to uncover the complex interaction networks that occur between organisms in nature. Interspecific communication between $S$. maltophilia and $P$. aeruginosa has been a subject of investigation in the last years, since these two bacterial species usually share ecological niches and, importantly, they are frequently coisolated in lungs of cystic fibrosis (CF) patients (Moskowitz et al., 2005). It has been reported that the DSF signal produced by $S$. maltophilia modulates $P$. aeruginosa behavior, including biofilm development and polymixin tolerance (Ryan et al., 2008) as well as virulence and persistence in lungs of CF patients (Twomey et al., 2012). However, as far as we know, the communication between these two potential human pathogens has been only studied in a unidirectional-way. We report here for the first time that $S$. maltophilia can also respond to signaling molecules produced by $P$. aeruginosa. In particular, we have shown that AHLs produced by $P$. aeruginosa stimulate swarming motility in S. maltophilia (Figure 4), a process in which SmoR might play a central role (Figure 5). Nevertheless, we cannot exclude that some other molecules present in the $P$. aeruginosa supernatant, such as rhamnolipids (Caiazza et al., 2005), could also participate in swarming stimulation. If so, it would explain the slightly stimulation observed in the $\Delta s m o R$ mutant, which perhaps could initiate its swarming motility due to the presence of rhamnolipids rather than AHL signals.

It is well established that complex population behaviors such as swarming motility are commonly regulated by AHL-QS systems (Daniels et al., 2004). Furthermore, previous studies have demonstrated that heterologous expression of lactonase AiiA from B. subtilis (Dong et al., 2000) reduces AHLs production and swarming motility in $P$. aeruginosa (Reimmann et al., 2002) and Burkholderia cepacia species (Wopperer et al., 2006). Regulation of bacterial motility by LuxR solos has been also reported. In Xoo, OryR positively regulates swimming and swarming motility by binding to the promoter of numerous flagella genes in response to plant signaling molecules (González et al., 2013).

To date, the only QS system described in S. maltophilia is DSF-QS, which is based on the signaling fatty acid molecule 11-cis-2-decenoic acid (Huang and Lee Wong, 2007; Huedo et al., 2014). Previous studies have evidenced that the DSF system regulates several virulence-related processes including bacterial motility, biofilm formation, antibiotic resistance and virulence (Fouhy et al., 2007; Deng et al., 2011; Huedo et al., 2014). In the related bacterium Burkholderia cenocepacia, both QS systems co-exist: the DSF -designated BDSF- (Boon et al., 2008; Deng et al., 2010) and the AHL (Wopperer et al., 2006) systems. Interestingly, it has been shown that certain biological functions such as motility, biofilm formation and virulence, are co-regulated by the BDSF- and AHL-dependent QS systems in $B$. cenocepacia (Deng et al., 2009, 2013). Although AHL production has not been reported in S. maltophilia, our findings suggest that, besides DSF, exogenous AHL signals could also regulate QS-related phenotypes-as observed for swarming motility-, by interacting with the LuxR solo SmoR.

\section{Acknowledgments}

This work has been supported in part by the Seventh Research Framework Programme of the European Union (HEALTH-F32009-223101), the Spanish MICINN (BFU2010-17199) and the Catalan AGAUR (2014SGR-1280).

\section{Supplementary Material}

The Supplementary Material for this article can be found online at: http://journal.frontiersin.org/article/10.3389/fcimb. 2015.00041/abstract 


\section{References}

Ahmer, B. M., van Reeuwijk, J., Timmers, C. D., Valentine, P. J., and Heffron, F. (1998). Salmonella typhimurium encodes an SdiA homolog, a putative quorum sensor of the LuxR family, that regulates genes on the virulence plasmid. J. Bacteriol. 180, 1185-1193.

Ahmer, B. M. M. (2004). Cell-to-cell signalling in Escherichia coli and Salmonella enterica. Mol. Microbiol. 52, 933-945. doi: 10.1111/j.1365-2958.2004.04054.x

Altschul, S. F., Gish, W., Miller, W., Myers, E. W., and Lipman, D. J. (1990). Basic local alignment search tool. J. Mol. Biol. 215, 403-410. doi: 10.1016/S00222836(05)80360-2

Altschul, S. F., Madden, T. L., Schäffer, A. A., Zhang, J., Zhang, Z., Miller, W., et al. (1997). Gapped BLAST and PSI-BLAST: a new generation of protein database search programs. Nucleic Acids Res. 25, 3389-3402. doi: 10.1093/nar/25.1 7.3389

Boon, C., Deng, Y., Wang, L.-H., He, Y., Xu, J.-L., Fan, Y., et al. (2008). A novel DSF-like signal from Burkholderia cenocepacia interferes with Candida albicans morphological transition. ISME J. 2, 27-36. doi: 10.1038/ismej.2007.76

Bottomley, M. J., Muraglia, E., Bazzo, R., and Carfi, A. (2007). Molecular insights into quorum sensing in the human pathogen Pseudomonas aeruginosa from the structure of the virulence regulator LasR bound to its autoinducer. J. Biol. Chem. 282, 13592-13600. doi: 10.1074/jbc.M700556200

Brameyer, S., Kresovic, D., Bode, H. B., and Heermann, R. (2015). Dialkylresorcinols as bacterial signaling molecules. Proc. Natl. Acad. Sci. U.S.A. 112, 572-577. doi: 10.1073/pnas. 1417685112

Brooke, J. S. (2012). Stenotrophomonas maltophilia: an emerging global opportunistic pathogen. Clin. Microbiol. Rev. 25, 2-41. doi: 10.1128/CMR.00019-11

Caiazza, N. C., Shanks, R. M. Q., and O’Toole, G. A. (2005). Rhamnolipids modulate swarming motility patterns of Pseudomonas aeruginosa. J. Bacteriol. 187, 7351-7361. doi: 10.1128/JB.187.21.7351-7361.2005

Chatterjee, J., Miyamoto, C. M., and Meighen, E. A. (1996). Autoregulation of luxR: the Vibrio harveyi lux-operon activator functions as a repressor. Mol. Microbiol. 20, 415-425. doi: 10.1111/j.1365-2958.1996.tb02628.x

Choi, K.-H., Kumar, A., and Schweizer, H. P. (2006). A 10-min method for preparation of highly electrocompetent Pseudomonas aeruginosa cells: application for DNA fragment transfer between chromosomes and plasmid transformation. J. Microbiol. Methods 64, 391-397. doi: 10.1016/j.mimet.2005.06.001

Choi, S. H., and Greenberg, E. P. (1991). The C-terminal region of the Vibrio fischeri LuxR protein contains an inducer-independent lux gene activating domain. Proc. Natl. Acad. Sci. U.S.A. 88, 11115-11119. doi: 10.1073/pnas.88.24.11115

Chugani, S., and Greenberg, E. P. (2014). An evolving perspective on the Pseudomonas aeruginosa orphan quorum sensing regulator QscR. Front. Cell. Infect. Microbiol. 452. doi: 10.3389/fcimb.2014.00152

Chugani, S. A., Whiteley, M., Lee, K. M., D’Argenio, D., Manoil, C., and Greenberg, E. P. (2001). QscR, a modulator of quorum-sensing signal synthesis and virulence in Pseudomonas aeruginosa. Proc. Natl. Acad. Sci. U.S.A. 98, 2752-2757. doi: 10.1073/pnas.051624298

Crossman, L. C., Gould, V. C., Dow, J. M., Vernikos, G. S., Okazaki, A., Sebaihia, M., et al. (2008). The complete genome, comparative and functional analysis of Stenotrophomonas maltophilia reveals an organism heavily shielded by drug resistance determinants. Genome Biol. 9:R74. doi: 10.1186/gb-2008-9-4-r74

Daniels, R., Vanderleyden, J., and Michiels, J. (2004). Quorum sensing and swarming migration in bacteria. FEMS Microbiol. Rev. 28, 261-289. doi: 10.1016/j.femsre.2003.09.004

Demko, C. A., Stern, R. C., and Doershuk, C. F. (1998). Stenotrophomonas maltophilia in cystic fibrosis: incidence and prevalence. Pediatr. Pulmonol. 25, 304-308.

Deng, Y., Boon, C., Eberl, L., and Zhang, L.-H. (2009). Differential modulation of Burkholderia cenocepacia virulence and energy metabolism by the quorumsensing signal BDSF and its synthase. J. Bacteriol. 191, 7270-7278. doi: 10.1128/JB.00681-09

Deng, Y., Lim, A., Wang, J., Zhou, T., Chen, S., Lee, J., et al. (2013). Cis2-dodecenoic acid quorum sensing system modulates $\mathrm{N}$-acyl homoserine lactone production through RpfR and cyclic di-GMP turnover in Burkholderia cenocepacia. BMC Microbiol. 13:148. doi: 10.1186/1471-2180-13-148
Deng, Y., Wu, J., Eberl, L., and Zhang, L.-H. (2010). Structural and functional characterization of diffusible signal factor family quorum-sensing signals produced by members of the Burkholderia cepacia complex. Appl. Environ. Microbiol. 76, 4675-4683. doi: 10.1128/AEM.00480-10

Deng, Y., Wu, J., Tao, F., and Zhang, L.-H. (2011). Listening to a new language: DSF-based quorum sensing in Gram-negative bacteria. Chem. Rev. 111, 160-173. doi: 10.1021/cr100354f

Devine, J. H., Shadel, G. S., and Baldwin, T. O. (1989). Identification of the operator of the lux regulon from the Vibrio fischeri strain ATCC7744. Proc. Natl. Acad. Sci. U.S.A. 86, 5688-5692. doi: 10.1073/pnas.86.15.5688

Dong, Y.-H., Xu, J.-L., Li, X.-Z., and Zhang, L.-H. (2000). AiiA, an enzyme that inactivates the acylhomoserine lactone quorum-sensing signal and attenuates the virulence of Erwinia carotovora. Proc. Natl. Acad. Sci. U.S.A. 97, 3526-3531. doi: $10.1073 /$ pnas.97.7.3526

Federle, M. J., and Bassler, B. L. (2003). Interspecies communication in bacteria. J. Clin. Invest. 112, 1291-1299. doi: 10.1172/JCI200320195

Ferluga, S., Bigirimana, J., Höfte, M., and Venturi, V. (2007). A LuxR homologue of Xanthomonas oryzae pv. oryzae is required for optimal rice virulence. Mol. Plant Pathol. 8, 529-538. doi: 10.1111/j.1364-3703.2007.00415.x

Ferluga, S., and Venturi, V. (2009). OryR is a LuxR-family protein involved in interkingdom signaling between pathogenic Xanthomonas oryzae pv. oryzae and rice. J. Bacteriol. 191, 890-897. doi: 10.1128/JB.01507-08

Ferrer-Navarro, M., Planell, R., Yero, D., Mongiardini, E., Torrent, G., Huedo, P., et al. (2013). Abundance of the quorum-sensing factor Ax21 in four strains of Stenotrophomonas maltophilia correlates with mortality rate in a new zebrafish model of infection. PLoS ONE 8:e67207. doi: 10.1371/journal.pone.0067207

Fouhy, Y., Scanlon, K., Schouest, K., Spillane, C., Crossman, L., Avison, M. B. et al. (2007). Diffusible signal factor-dependent cell-cell signaling and virulence in the nosocomial pathogen Stenotrophomonas maltophilia. J. Bacteriol. 189, 4964-4968. doi: 10.1128/JB.00310-07

Fried, L., Lassak, J., and Jung, K. (2012). A comprehensive toolbox for the rapid construction of lacZ fusion reporters. J. Microbiol. Methods 91, 537-543. doi: 10.1016/j.mimet.2012.09.023

Fuqua, C., and Greenberg, E. P. (2002). Listening in on bacteria: acyl-homoserine lactone signalling. Nat. Rev. Mol. Cell Biol. 3, 685-695. doi: 10.1038/nrm907

Fuqua, C., Winans, S. C., and Greenberg, E. P. (1996). Census and consensus in bacterial ecosystems: the LuxR-LuxI family of quorumsensing transcriptional regulators. Annu. Rev. Microbiol. 50, 727-751. doi: 10.1146/annurev.micro.50.1.727

Fuqua, C. (2006). The QscR quorum-sensing regulon of Pseudomonas aeruginosa: an orphan claims its identity. J. Bacteriol. 188, 3169-3171. doi: 10.1128/JB.188.9.3169-3171.2006

Fuqua, W. C., and Winans, S. C. (1994). A LuxR-LuxI type regulatory system activates Agrobacterium Ti plasmid conjugal transfer in the presence of a plant tumor metabolite. J. Bacteriol. 176, 2796-2806.

Fuqua, W. C., Winans, S. C., and Greenberg, E. P. (1994). Quorum sensing in bacteria: the LuxR-LuxI family of cell density-responsive transcriptional regulators. J. Bacteriol. 176, 269-275.

González, J. F., Myers, M. P., and Venturi, V. (2013). The inter-kingdom solo OryR regulator of Xanthomonas oryzae is important for motility. Mol. Plant Pathol. 14, 211-221. doi: 10.1111/j.1364-3703.2012.00843.x

Hanzelka, B. L., and Greenberg, E. P. (1995). Evidence that the N-terminal region of the Vibrio fischeri LuxR protein constitutes an autoinducer-binding domain. J. Bacteriol. 177, 815-817.

Hoang, T. T., Karkhoff-Schweizer, R. R., Kutchma, A. J., and Schweizer, H. P. (1998). A broad-host-range Flp-FRT recombination system for sitespecific excision of chromosomally-located DNA sequences: application for isolation of unmarked Pseudomonas aeruginosa mutants. Gene 212, 77-86. doi: 10.1016/S0378-1119(98)00130-9

Huang, T.-P., and Lee Wong, A. C. (2007). Extracellular fatty acids facilitate flagella-independent translocation by Stenotrophomonas maltophilia. Res. Microbiol. 158, 702-711. doi: 10.1016/j.resmic.2007.09.002

Hudaiberdiev, S., Choudhary, K. S., Vera Alvarez, R., Gelencsér, Z., Ligeti, B., Lamba, D., et al. (2015). Census of solo LuxR genes in prokaryotic genomes. Front. Cell. Infect. Microbiol. 5:4. doi: 10.3389/fcimb.2015.00004

Huedo, P., Yero, D., Martínez-Servat, S., Estibariz, I., Planell, R., Martínez, P., et al. (2014). Two different rpf clusters distributed among a population of Stenotrophomonas maltophilia clinical strains display differential diffusible 
signal factor production and virulence regulation. J. Bacteriol. 196, 2431-2442. doi: 10.1128/JB.01540-14

Jacobs, M. A., Alwood, A., Thaipisuttikul, I., Spencer, D., Haugen, E., Ernst, S., et al. (2003). Comprehensive transposon mutant library of Pseudomonas aeruginosa. Proc. Natl. Acad. Sci. 100, 14339-14344. doi: 10.1073/pnas.2036282100

Kearns, D. B. (2010). A field guide to bacterial swarming motility. Nat. Rev. Microbiol. 8, 634-644. doi: 10.1038/nrmicro2405

Koch, B., Liljefors, T., Persson, T., Nielsen, J., Kjelleberg, S., and Givskov, M. (2005). The LuxR receptor: the sites of interaction with quorum-sensing signals and inhibitors. Microbiology 151, 3589-3602. doi: 10.1099/mic.0.27954-0

Kovach, M. E., Elzer, P. H., Hill, D. S., Robertson, G. T., Farris, M. A., Roop, R. M., et al. (1995). Four new derivatives of the broad-host-range cloning vector pBBR1MCS, carrying different antibiotic-resistance cassettes. Gene 166, 175-176. doi: 10.1016/0378-1119(95)00584-1

Lee, J.-H., Lequette, Y., and Greenberg, E. P. (2006). Activity of purified QscR, a Pseudomonas aeruginosa orphan quorum-sensing transcription factor. Mol. Microbiol. 59, 602-609. doi: 10.1111/j.1365-2958.2005.04960.x

Lequette, Y., Lee, J.-H., Ledgham, F., Lazdunski, A., and Greenberg, E. P. (2006). A distinct QscR regulon in the Pseudomonas aeruginosa quorum-sensing circuit. J. Bacteriol. 188, 3365-3370. doi: 10.1128/JB.188.9.3365-3370.2006

Letunic, I., Doerks, T., and Bork, P. (2009). SMART 6: recent updates and new developments. Nucleic Acids Res. 37, D229-232. doi: 10.1093/nar/gkn808

Malki, A., Caldas, T., Abdallah, J., Kern, R., Eckey, V., Kim, S. J., et al. (2005). Peptidase activity of the Escherichia coli Hsp31 chaperone. J. Biol. Chem. 280, 14420-14426. doi: 10.1074/jbc.M408296200

Malki, A., Kern, R., Abdallah, J., and Richarme, G. (2003). Characterization of the Escherichia coli YedU protein as a molecular chaperone. Biochem. Biophys. Res. Commun. 301, 430-436. doi: 10.1016/S0006-291X(02)03053-X

Maurhofer, M., Reimmann, C., Schmidli-Sacherer, P., Heeb, S., Haas, D., and Défago, G. (1998). Salicylic acid biosynthetic genes expressed in pseudomonas fluorescens strain P3 improve the induction of systemic resistance in tobacco against tobacco necrosis virus. Phytopathology 88, 678-684. doi: 10.1094/PHYTO.1998.88.7.678

Michael, B., Smith, J. N., Swift, S., Heffron, F., and Ahmer, B. M. (2001). SdiA of Salmonella enterica is a LuxR homolog that detects mixed microbial communities. J. Bacteriol. 183, 5733-5742. doi: 10.1128/JB.183.19.57335742.2001

Miller, J. H. (1972). Experiments in Molecular Genetics. New York, NY: Cold Spring Harbor Laboratory.

Miller, M. B., and Bassler, B. L. (2001). Quorum sensing in bacteria. Annu. Rev. Microbiol. 55, 165-199. doi: 10.1146/annurev.micro.55.1.165

Minogue, T. D., Wehland-von Trebra, M., Bernhard, F., and von Bodman, S. B. (2002). The autoregulatory role of EsaR, a quorum-sensing regulator in Pantoea stewartii ssp. stewartii: evidence for a repressor function. Mol. Microbiol. 44, 1625-1635. doi: 10.1046/j.1365-2958.2002.02987.x

Mitchell, A., Chang, H.-Y., Daugherty, L., Fraser, M., Hunter, S., Lopez, R., et al. (2014). The InterPro protein families database: the classification resource after 15 years. Nucleic Acids Res. 43, D213-D221. doi: 10.1093/nar/gku1243

Moskowitz, S. M., Gibson, R. L., and Effmann, E. L. (2005). Cystic fibrosis lung disease: genetic influences, microbial interactions, and radiological assessment. Pediatr. Radiol. 35, 739-757. doi: 10.1007/s00247-005-1445-3

Mujacic, M., Bader, M. W., and Baneyx, F. (2004). Escherichia coli Hsp31 functions as a holding chaperone that cooperates with the DnaK-DnaJ-GrpE system in the management of protein misfolding under severe stress conditions. Mol. Microbiol. 51, 849-859. doi: 10.1046/j.1365-2958.2003.03871.x

Mujacic, M., and Baneyx, F. (2006). Regulation of Escherichia coli hchA, a stressinducible gene encoding molecular chaperone Hsp31. Mol. Microbiol. 60, 1576-1589. doi: 10.1111/j.1365-2958.2006.05207.x

Mujacic, M., and Baneyx, F. (2007). Chaperone Hsp31 contributes to acid resistance in stationary-phase Escherichia coli. Appl. Environ. Microbiol. 73, 1014-1018. doi: 10.1128/AEM.02429-06

Oinuma, K.-I., and Greenberg, E. P. (2011). Acyl-Homoserine lactone binding to and stability of the orphan Pseudomonas aeruginosa Quorum-Sensing Signal Receptor QscR. J. Bacteriol. 193, 421-428. doi: 10.1128/JB.01041-10

Overhage, J., Lewenza, S., Marr, A. K., and Hancock, R. E. W. (2007). Identification of genes involved in swarming motility using a Pseudomonas aeruginosa PAO1 mini-Tn5-lux mutant library. J. Bacteriol. 189, 2164-2169. doi: 10.1128/JB.01623-06
Patankar, A. V., and González, J. E. (2009). Orphan LuxR regulators of quorum sensing. FEMS Microbiol. Rev. 33, 739-756. doi: 10.1111/j.15746976.2009.00163.x

Reimmann, C., Ginet, N., Michel, L., Keel, C., Michaux, P., Krishnapillai, V., et al. (2002). Genetically programmed autoinducer destruction reduces virulence gene expression and swarming motility in Pseudomonas aeruginosa PAO1. Microbiology 148, 923-932.

Ryan, R. P., and Dow, J. M. (2008). Diffusible signals and interspecies communication in bacteria. Microbiology 154, 1845-1858. doi: 10.1099/mic.0.2008/017871-0

Ryan, R. P., Fouhy, Y., Garcia, B. F., Watt, S. A., Niehaus, K., Yang, L., et al. (2008). Interspecies signalling via the Stenotrophomonas maltophilia diffusible signal factor influences biofilm formation and polymyxin tolerance in Pseudomonas aeruginosa. Mol. Microbiol. 68, 75-86. doi: 10.1111/j.1365-2958.2008.0 6132.x

Sambrook, J., Fritsch, E. F., and Maniatis, T. (1989). Molecular Cloning: A Laboratory Manual. Cold Spring Harbor, NY: Cold Spring Harbor Laboratory Press.

Sastry, M. S. R., Korotkov, K., Brodsky, Y., and Baneyx, F. (2002). Hsp31, the Escherichia coli yedU gene product, is a molecular chaperone whose activity is inhibited by ATP at high temperatures. J. Biol. Chem. 277, 46026-46034. doi: 10.1074/jbc.M205800200

Shadel, G. S., and Baldwin, T. O. (1992). Positive autoregulation of the Vibrio fischeri luxR gene. LuxR and autoinducer activate cAMP-catabolite gene activator protein complex-independent and -dependent luxR transcription. J. Biol. Chem. 267, 7696-7702.

Shadel, G. S., Young, R., and Baldwin, T. O. (1990). Use of regulated cell lysis in a lethal genetic selection in Escherichia coli: identification of the autoinducer-binding region of the LuxR protein from Vibrio fischeri ATCC 7744. J. Bacteriol. 172, 3980-3987.

Shaw, P. D., Ping, G., Daly, S. L., Cha, C., Cronan, J. E., Rinehart, K. L., et al. (1997). Detecting and characterizing $\mathrm{N}$-acyl-homoserine lactone signal molecules by thin-layer chromatography. Proc. Natl. Acad. Sci. U.S.A. 94, 6036-6041. doi: 10.1073/pnas.94.12.6036

Shiba, T., Ishiguro, K., Takemoto, N., Koibuchi, H., and Sugimoto, K. (1995). Purification and characterization of the Pseudomonas aeruginosa $\mathrm{NfxB}$ protein, the negative regulator of the $\mathrm{nfxB}$ gene. J. Bacteriol. 177, 5872-5877.

Slock, J., VanRiet, D., Kolibachuk, D., and Greenberg, E. P. (1990). Critical regions of the Vibrio fischeri luxR protein defined by mutational analysis. J. Bacteriol. 172, 3974-3979.

Stevens, A. M., and Greenberg, E. P. (1997). Quorum sensing in Vibrio fischeri: essential elements for activation of the luminescence genes. J. Bacteriol. 179, 557-562.

Subedi, K. P., Choi, D., Kim, I., Min, B., and Park, C. (2011). Hsp31 of Escherichia coli K-12 is glyoxalase III. Mol. Microbiol. 81, 926-936. doi: 10.1111/j.13652958.2011.07736.x

Subramoni, S., and Venturi, V. (2009a). LuxR-family "solos": bachelor sensors/regulators of signalling molecules. Microbiol. Read. Engl. 155, 1377-1385. doi: 10.1099/mic.0.026849-0

Subramoni, S., and Venturi, V. (2009b). PpoR is a conserved unpaired LuxR solo of Pseudomonas putida which binds N-acyl homoserine lactones. BMC Microbiol. 9:125. doi: 10.1186/1471-2180-9-125

Tamura, K., Stecher, G., Peterson, D., Filipski, A., and Kumar, S. (2013). MEGA6: Molecular Evolutionary Genetics Analysis version 6.0. Mol. Biol. Evol. 30, 2725-2729. doi: 10.1093/molbev/mst197

Thomas-Chollier, M., Defrance, M., Medina-Rivera, A., Sand, O., Herrmann, C., Thieffry, D., et al. (2011). RSAT 2011: regulatory sequence analysis tools. Nucleic Acids Res. 39, W86-W91. doi: 10.1093/nar/ $\operatorname{gkr} 377$

Twomey, K. B., O’Connell, O. J., McCarthy, Y., Dow, J. M., O’Toole, G. A., Plant, B. J., et al. (2012). Bacterial cis-2-unsaturated fatty acids found in the cystic fibrosis airway modulate virulence and persistence of Pseudomonas aeruginosa. ISME J. 6, 939-950. doi: 10.1038/ismej.2011.167

Vannini, A., Volpari, C., Gargioli, C., Muraglia, E., Cortese, R., Francesco, R D., et al. (2002). The crystal structure of the quorum sensing protein TraR bound to its autoinducer and target DNA. EMBO J. 21, 4393-4401. doi: 10.1093/emboj/cdf459 
Waters, C. M., and Bassler, B. L. (2005). Quorum sensing: cell-to-cell communication in bacteria. Annu. Rev. Cell Dev. Biol. 21, 319-346. doi: 10.1146/annurev.cellbio.21.012704.131001

Whitehead, N. A., Barnard, A. M., Slater, H., Simpson, N. J., and Salmond, G. P. (2001). Quorum-sensing in Gram-negative bacteria. FEMS Microbiol. Rev. 25, 365-404. doi: 10.1111/j.1574-6976.2001.tb00583.x

Wopperer, J., Cardona, S. T., Huber, B., Jacobi, C. A., Valvano, M. A., and Eberl, L. (2006). A quorum-quenching approach to investigate the conservation of quorum-sensing-regulated functions within the Burkholderia cepacia Complex. Appl. Environ. Microbiol. 72, 1579-1587. doi: 10.1128/AEM.72.2.15791587.2006

Yao, Y., Martinez-Yamout, M. A., Dickerson, T. J., Brogan, A. P., Wright, P. E., and Dyson, H. J. (2006). Structure of the Escherichia coli quorum sensing protein SdiA: activation of the folding switch by acyl homoserine lactones. J. Mol. Biol. 355, 262-273. doi: 10.1016/j.jmb.2005.10.041

Zhang, R., Pappas, T., Brace, J. L., Miller, P. C., Oulmassov, T., Molyneaux, J. M., et al. (2002). Structure of a bacterial quorum-sensing transcription factor complexed with pheromone and DNA. Nature 417, 971-974. doi: 10.1038 /nature00833

Zhu, J., Chai, Y., Zhong, Z., Li, S., and Winans, S. C. (2003). Agrobacterium bioassay strain for ultrasensitive detection of N-Acylhomoserine lactone-type quorum-sensing molecules: detection of autoinducers in
Mesorhizobium huakuii. Appl. Environ. Microbiol. 69, 6949-6953. doi: 10.1128/AEM.69.11.6949-6953.2003

Zhu, J., and Winans, S. C. (1999). Autoinducer binding by the quorum-sensing regulator TraR increases affinity for target promoters in vitro and decreases TraR turnover rates in whole cells. Proc. Natl. Acad. Sci. U.S.A. 96, 4832-4837. doi: $10.1073 /$ pnas.96.9.4832

Zhu, J., and Winans, S. C. (2001). The quorum-sensing transcriptional regulator TraR requires its cognate signaling ligand for protein folding, protease resistance, and dimerization. Proc. Natl. Acad. Sci. U.S.A. 98, 1507-1512. doi: 10.1073/pnas.98.4.1507

Conflict of Interest Statement: The authors declare that the research was conducted in the absence of any commercial or financial relationships that could be construed as a potential conflict of interest.

Copyright (c) 2015 Martínez, Huedo, Martínez-Servat, Planell, Ferrer-Navarro, Daura, Yero and Gibert. This is an open-access article distributed under the terms of the Creative Commons Attribution License (CC BY). The use, distribution or reproduction in other forums is permitted, provided the original author (s) or licensor are credited and that the original publication in this journal is cited, in accordance with accepted academic practice. No use, distribution or reproduction is permitted which does not comply with these terms. 